\title{
Cluster Reception of DVB-S2 Signals for Mobile Terminals
}

\author{
Luca Simone Ronga \\ CNIT - University of Florence \\ Florence, Italy \\ Email: luca.ronga@cnit.it
}

\author{
Enrico Del Re \\ DET - University of Florence \\ Florence, Italy \\ Email: enrico.delre@unifi.it
}

\author{
Fabrizio Gandon \\ DET - University of Florence \\ Florence, Italy
}

\begin{abstract}
The problem addressed in this work is the direct reception of DVB-S(2) satellite signals from mobile terminals equipped with low gain (non directive) antennas. DVB-S2 is broadcasted with GEO satellites and mobile receivers usually need great antenna gains in order to compensate the large pathloss encountered in the downlink. Cooperation strategies can help in this context. In the paper we show analytical and simulated performances of a physical level link cooperation conducted by a cluster of mobile users in the vicinity of the current active user.
\end{abstract}

\section{INTRODUCTION}

DVB-S(2) is the second generation system for Broadcasting, Interactive Services, News Gathering and other broadband satellite applications [1], [2]. This system gets advantages from the most recent developments of channel coding LDPC, joined with several modulation orders (QPSK, 8-PSK, 16-APSK and 32-APSK). The possibility to change the modulation and coding parameters for each frame (VCM) and the ability to change these parameters according to the channel (ACM), are the main new system characteristics.

Direct reception of DVB-S2 satellite signals from mobile terminals, equipped with non directive antennas, is becoming of great interest among manufacturers and operators. Low orbit constellations are technically preferred for mobile terminal reception due to the reduced path loss. Economical issues however, have recently redirected the interest to medium and geostationary constellations, eventually assisted by high altitude platforms. Since the satellite power is limited by technology and the maximum allowable mass of satellites, downlink EIRP is a limited resource which can be increased at the expense of coverage, by reducing the spot dimensions [3]. Even in the latter case, a sufficient $C / N$ value cannot be reached by the receiver handset for the correct reception of the DVB-S(2) downstream. Recently, a new class of methods called cooperative communication has been proposed [4], [5], [6], that enables single-antenna mobiles in a multi-user environment to share their antennas and generate a virtual multiple-antenna transmitter that allows them to achieve transmit/receive diversity. The mobile wireless channel suffers from fading, meaning that the signal attenuation can vary significantly over the course of a given transmission. Transmitting/receiving independent copies of the signal generates diversity and can effectively combat the deleterious

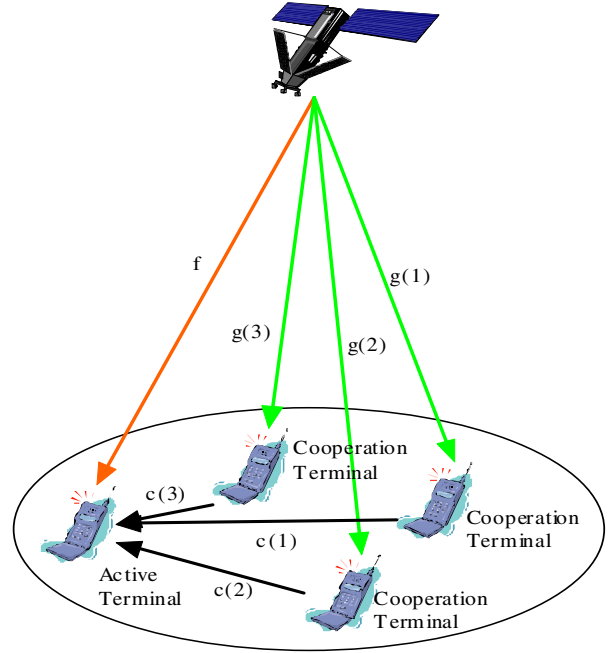

Fig. 1. Downlink Satellite Cooperation Scenario

effects of fading. In particular, spatial diversity is generated by transmitting/receiving signals from different locations, thus allowing independently faded versions of the signal at the receiver. Cooperative communication generates this diversity in a new and interesting way.

The main cooperation strategies are Detect and Forward [4], [5], Amplify and Forward [6] and Selective Forward [7]. The considered cooperation scheme in this paper is Amplify and Forward (AF) [8].

\section{System Model}

The adopted cooperation scenario is depicted in Figure 1. The main operating parameters are reported in table I.

The basic idea of AF strategy [8] is that around a given terminal, there can be other single-antenna terminals which can be used to enhance diversity by forming a virtual (or distributed) multiantenna system (see Figure 1) where the satellite signal is received from the active terminal and a number of cooperating relays. The cooperating terminals retransmit the received signal after amplification. The AF strategy is particularly efficient when the cooperating terminals are located close to the active one so that the cooperative links $(c(1), c(2), c(3))$ are characterized by high signal-to-noise ratios and the link from the satellite to the active terminal $(f)$ is 


\begin{tabular}{lllr}
\hline$d_{\text {sat }}$ & 36000 & {$[\mathrm{~km}]$} & satellite terminal distance \\
$d_{\text {coop }}$ & 10 & {$[\mathrm{~km}]$} & cooperative terminal \\
$L_{\text {sat }}$ & -205.34 & {$[\mathrm{~dB}]$} & satellite terminal path loss \\
$L_{\text {coop }}$ & -118.5 & {$[\mathrm{~dB}]$} & cooperative terminal path loss \\
$B_{\text {sat }}$ & 36 & {$[\mathrm{MHz}]$} & transpoder bandwidth \\
$P_{\text {sat }}$ & 70 & {$[\mathrm{dBW}]$} & satellite power \\
$P_{\max }$ & 250 & {$[\mathrm{~mW}]$} & cooperative terminal maximum power \\
$G / T_{R x}$ & -24 & {$[\mathrm{~dB} / \mathrm{K}]$} & handheld receiver $G / T$ \\
$T_{\text {sys }}$ & 290 & {$[\mathrm{~K}]$} & system temperature \\
$F_{c}$ & 2000 & {$[\mathrm{MHz}]$} & cooperation channel frequency \\
$F_{d}$ & 11750 & {$[\mathrm{MHz}]$} & downlink channel frequency \\
\hline
\end{tabular}

TABLE I

MAIN OPERATIONAL PARAMETERS

comparable with the links from the satellite to cooperating devices. AF requires minimal processing at the cooperating terminal but it needs a consistent storage capability of the analog received signal. As in [8] we consider the amplification factor $A$ relationship given by

$$
A_{i}^{2}=\frac{P_{\max }}{P_{\text {sat }}|g(i)|^{2}+N}
$$

where $P_{\text {sat }}$ is the satellite downlink power and $P_{\max }$ the cooperative terminal maximum power; $M$ is the number of cooperating terminals, $g(i)$ the $i$-th link pathloss, $N=$ $K T s y s B_{\text {sat }}$ the noise spectral density at the earth terminals (see table I). With this choice we obtain an expression of the resulting $C / N$ on the active terminal.

$$
\frac{C}{N}=\gamma_{f}+\sum_{i=1}^{M} \frac{\gamma_{g_{i}} \gamma_{c_{i}}}{\gamma_{g_{i}}+\gamma_{c_{i}}+1}
$$

By assuming that all of the cooperating terminals have the same characteristics and the cooperative channels are similar we can simplify the previous expression in

$$
\frac{C}{N}=\gamma_{f}+M \frac{\gamma_{g} \gamma_{c}}{\gamma_{g}+\gamma_{c}+1}
$$

furthermore we can consider $\gamma_{f}=\gamma_{g}$ so the variables concerning the channel become two $\left(\gamma_{f}\right.$ and $\left.\gamma_{c}\right)$.

$$
\frac{C}{N}=\gamma_{f}\left(1+M \frac{\gamma_{c}}{1+\gamma_{f}+\gamma_{c}}\right)
$$

The previous expression becomes (see Appendix B)

$$
\frac{C}{N}=\frac{P_{\text {sat }}|f|^{2}}{N}\left(1+M \frac{A^{2}|c|^{2}}{1+A^{2}|c|^{2}}\right)
$$

thus the signal-to-noise ratio depends on $\gamma_{z}=$ $f\left(P_{\text {sat }}, A, M, f, c, N\right)$.

\section{LINK BUDGET CONSIDERATION}

As we can see in (5), AF cooperation can provide some advantages:

- $C / N$ improvement at $\mathrm{M}$ growth with all other parameters fixed;

- $C / N$ improvement depending on the choice of $A$ and $P_{\text {sat }}$ with $M, d_{\text {coop }}$ and $F_{c}$ fixed (see Figure 2);

- $C / N$ improvement with variable $L_{\text {coop }}$ and $M$ with $P_{\text {sat }}$ and $A$ fixed (see Figure 3);

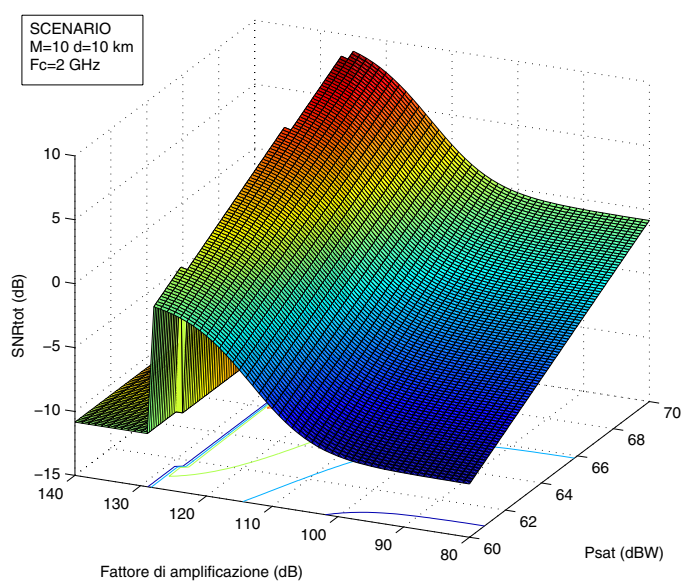

Fig. 2. Receiver SNR vs cooperative terminal amplification factor $(A)$ and satellite tx power $\left(P_{\text {sat }}\right)$

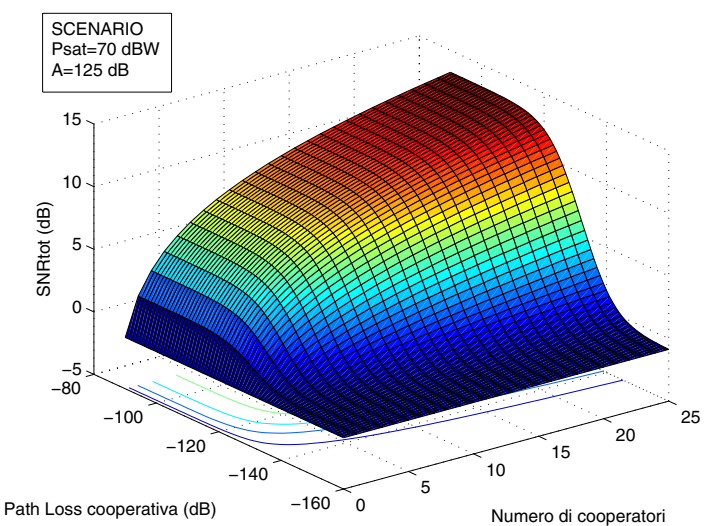

Fig. 3. Receiver SNR vs cooperation link loss $\left(L_{c o o p}\right)$ and number of cooperating terminals $(M)$

- $P_{\text {sat }}$ decreasing (spot area coverage expansion) at $M$ growth for a fixed $C / N$;

The target is to try to get a value of the (5) such to guarantee the fruition of the standard DVB-S2 services, a fact that was not realizable using only one mobile. Figure 2 shows the limit of $C / N$ improvement due to choice not to sorpass the $P_{\max }$ constraint

$$
A^{2} \leq \frac{P_{\max }}{P_{\text {sat }}|f|^{2}+N}
$$

and the amplification factor range where it is convenient to work to obtain performances gain $(A \approx 110-125 \mathrm{~dB})$. We chose $M=10, d_{\text {coop }}=10 \mathrm{~km}$ and $F_{c}=2 \mathrm{GHz}$ as fixed variables. That amplification factor range depends on the quality of cooperative links and it assume lower values decreasing $L_{\text {coop }}$. This last dependence is better shown in Figure 3, where the amplification factor is set to its maximum allowable value not violating the $P_{\max }$ constraint. In this figure we can notice the $C / N$ improvement as $M$ and $L_{\text {coop }}$ decrease. The required $C / N$ for the transmission modes in 
DVB-S2 standard [2] are reported in table II. As we can see,

\begin{tabular}{|l|c|c|r|}
\hline Modulation & useful Mb/s & Eb/No $(\mathbf{d B})$ & $\mathbf{C} / \mathbf{N}(\mathbf{d B})$ \\
\hline \hline QPSK 1/2 & 7.2 & 1.05 & 0.08 \\
QPSK 2/3 & 9.52 & 1.89 & 2.13 \\
QPSK 3/4 & 10.71 & 2.31 & 3.07 \\
QPSK 5/6 & 11.91 & 2.99 & 4.21 \\
QPSK 8/9 & 12.72 & 3.73 & 5.23 \\
8-PSK 2/3 & 14.26 & 3.65 & 5.65 \\
8-PSK 3/4 & 16.04 & 4.43 & 6.94 \\
8-PSK 5/6 & 17.85 & 5.41 & 8.38 \\
16-APSK 3/4 & 21.36 & 5.49 & 9.24 \\
16-APSK 4/5 & 22.79 & 6.03 & 10.07 \\
16-APSK 5/6 & 23.76 & 6.42 & 10.63 \\
\hline
\end{tabular}

TABLE II

REQUIRED $C / N$ WITH 7.2 MBAUd IN DOWNLINK

the AF cooperation strategy with $A=125 \mathrm{~dB}, P_{\text {sat }}=70$ $\mathrm{dBW}, B_{\text {sat }}=9 \mathrm{MHz}$ gives the chance to use the modulations QPSK, 8-PSK and 16-APSK in the downlink (the required values are under the surface of Figure 3 ). So for a given configuration of cooperators (link quality $L_{\text {coop }}$ and number $M)$ a specific subset of DVB-S2 compliant modulations ca be adopted.

All the results in this section have been derived from theoretical considerations. In particular we considered AWGN satellite channel and the coefficients $f$ and $g$ representing the satellite and cooperation path losses. In the next section a more realistic scenario is considered, with a cluster of satellite terminals with channels modeled with Corazza-Vatalaro model [9], [10], [11].

\section{Cluster Performance}

In the cooperative system model depicted in Figure 4 a DVB-S2 hub processes and sends the digital signals to a satellite A mobile DVB-S2 receiver (the active terminal) combines the signals coming from the satellite and from several mobile cooperators (as in the downlink model). The satellite-earth link is modeled with a Corazza-Vatalaro process while the cooperator-receiver link is represented only by an AWGN (the cooperation link channel model in the figure). The cooperative link path-loss value in the model is $118 \mathrm{~dB}$, correspondent to a cooperation frequency $F_{c}=2 G h z$ and a cooperation distance $d_{c}=10 \mathrm{~km}$.

The fading effect on the cooperative links due to terrestrial propagation has been considered of a limited impact on the overall performances due to the limited size of the cooperation cluster (within $10 \mathrm{~km}$ ), and it is included in CorazzaVatalaro downlink channel shadowing. A set of performances simulations has been conducted by setting lower values of the Rice factor for cooperation channels, to take into account the additional degradation of the cooperative signal chain. We also considered a cooperative channel estimation for the active terminal for carrier phase acquisition and tracking. An ideal channel acquisition and tracking has been considered in the current presentation of the work. The model has been simulated with a time resolution equal to $1 / 2 B_{\text {sat }}=1 / 14.8 \mathrm{M} \mathrm{Hz}$, with $B_{\text {sat }}$ being the bandwidth of the modulated QPSK signal

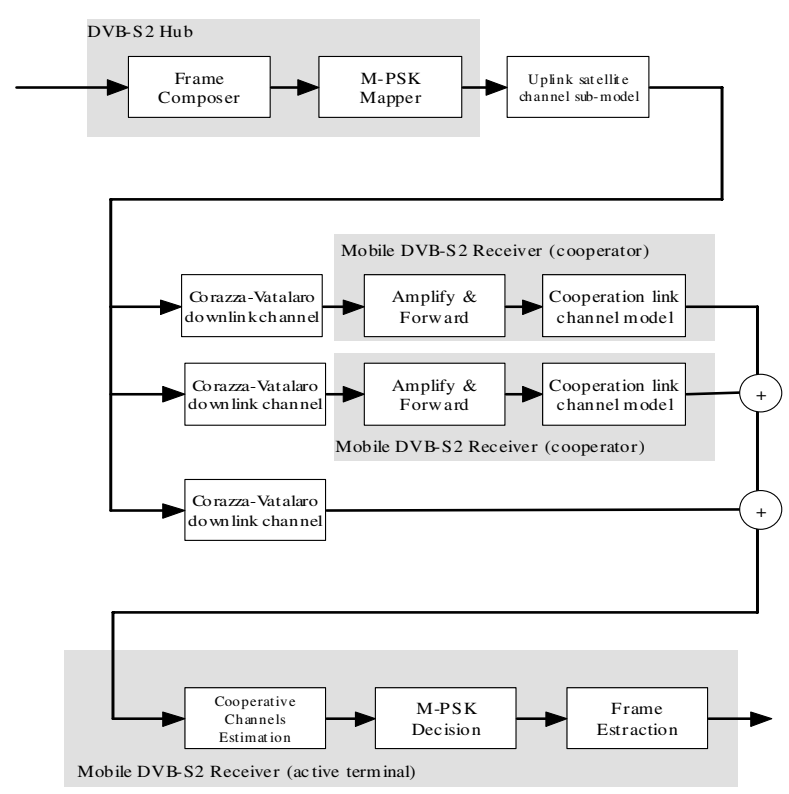

Fig. 4. Cooperative system model

$(F E C=1 / 2)$ considering an useful data rate of $7.2 \mathrm{Mb} / \mathrm{s}$ (TableII). The resulting BER versus $E_{b} / N_{o}$ curves for different configurations have been plotted.

The first graph in figure 5 shows the performances of QPSK and 8-PSK modulations with a Corazza-Vatalaro channel characterized by a Rice factor $R=20$.

The performances show an significant improvement, in terms of error probability, in comparison to the case in absence of cooperation for the same modulations (QPSK and 8PSK). QPSK shows a $B E R=10^{-4}$ for $E_{b} / N_{0}=20 d B$, while for the 8PSK gives a $B E R=10^{-3}$ to parity of $E_{b} / N_{0}$. Moreover 8PSK performances become sensibly worse with smaller values of $\mathrm{R}$ due to the reduction of the deterministic component of the ricean channel which result in heavy fluctuations of the signal. In the graph of figure 6 three conditions of shadowing are considered:

- $\mathrm{R}=20$ correspondent to very light shadowing values;

- $\mathrm{R}=15$ representing an intermediate value;

- $\mathrm{R}=10$ with significant shadowing values.

The curves of figure 7 show the advantages deriving from the use of the cooperation AF strategy considering the QPSK modulation. We can see how the performances improve as the number of cooperators increase: on the top of the figure is represented the situation in the absence of relays, then follow the performances with 5,10 and 15 cooperators. The comparison has been issued choosing a Rice factor $R=1$; the results $\left(B E R<10^{-2}\right)$ are acceptable for the channel coding techniques present in the DVB-S2 standard.

By varying the Rice factor $\mathrm{R}$ we obtain the results shown in figure 8 where QPSK performances with heavy shadowing ( $R=0.6)$, medium shadowing $(R=1)$ and light shadowing $(R=4)$ are compared. For $\mathrm{R}=4$ the performances are close to the target $\left(B E R=10^{-4}\right)$, while for $R=0.6$ the BER values 


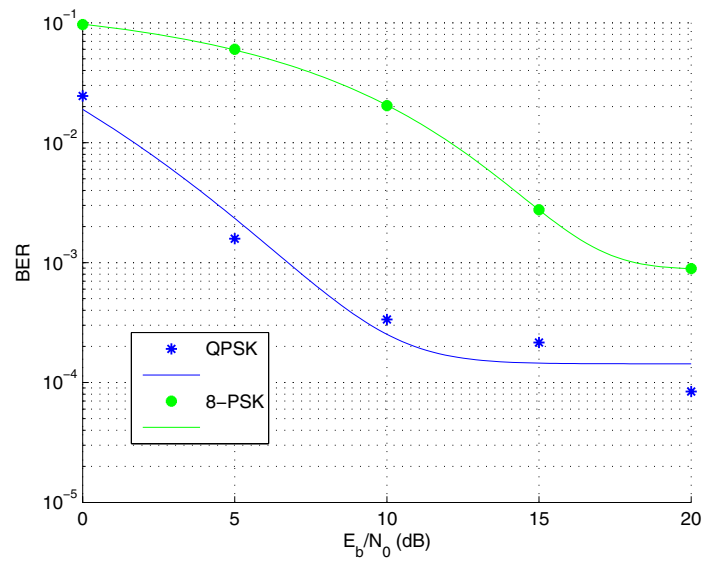

Fig. 5. QPSK and 8PSK with Rice factor $\mathrm{K}=20$ and 10 cooperators

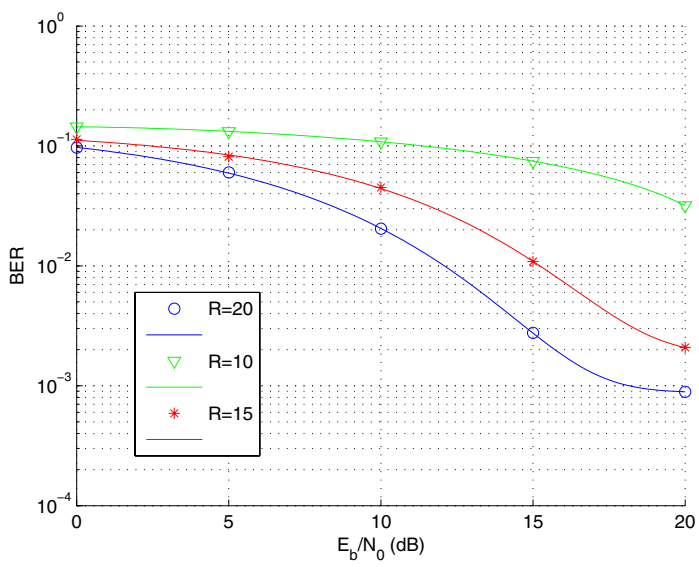

Fig. 6. $8 \mathrm{PSK}$ with variable $\mathrm{R}=10 ; 15 ; 20$ and 10 cooperators

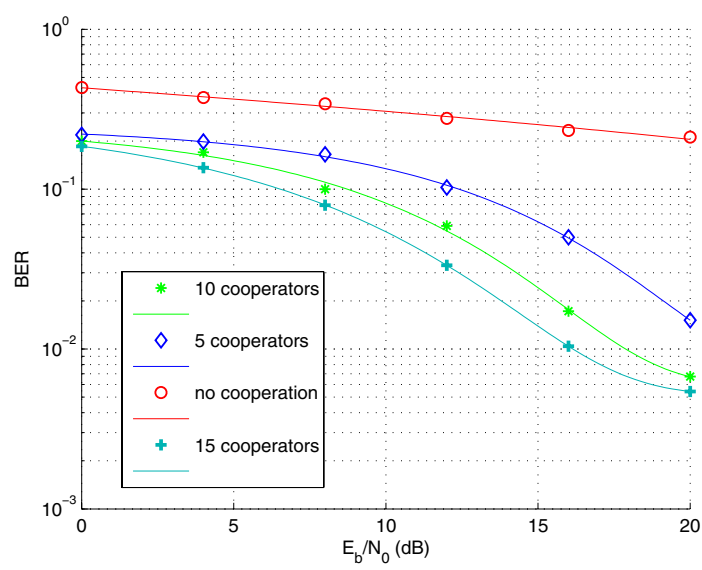

Fig. 7. QPSK with cooperator number variable $M=0 ; 5 ; 10 ; 15$ with $\mathrm{R}=1$

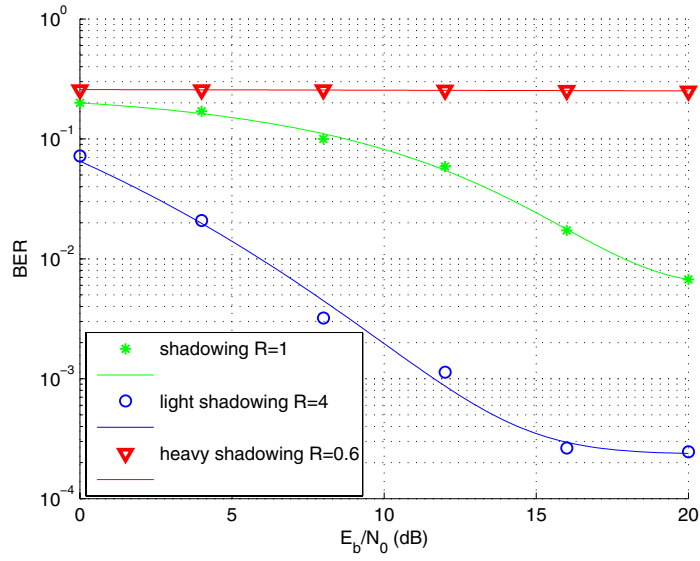

Fig. 8. QPSK for variable $\mathrm{R}=0.6 ; 1 ; 4$ and 10 cooperators

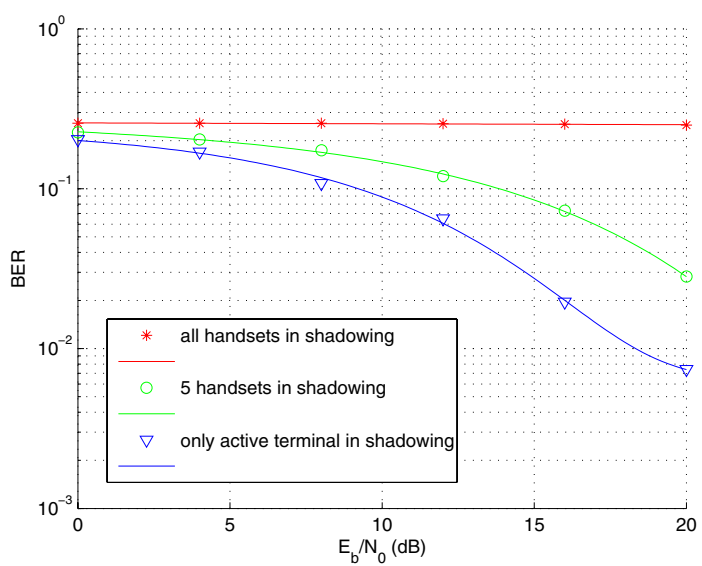

Fig. 9. QPSK varying handset number in shadowing for $\mathrm{R}=0.6$

are higher then target resulting unacceptable for DVB-S2.

It is worth noting that in these simulations all the handset share the same Rice factor $R$, modeling the situation where the consumers cooperators all work under homogeneous operational conditions. By considering a less critical situation, where only a subset of cooperating terminals are subject to heavy shadowing, we can see (figure 9) that the performances improve. Figure 9 shows the BER in the case of $50 \%$ of the handset are in heavy shadowing $(R=0.6)$ while the remaining ones have $R=1$.

Another comparison has been issued in the case of a degraded cooperative link, modeling the additional fading effect on the terrestrial cooperator-to-active-user channel. The performances for a QPSK modulation under these conditions are represented in Figure 10, where the effects of the terrestrial link are shown for a light $\left(R_{c}=10\right)$ and moderate $\left(R_{c}=4\right)$ shadowing environment.

\section{CONCLusions}

This paper shows a possible solution to the problem of the extension to the mobility (direct receipt on mobile terminals, 


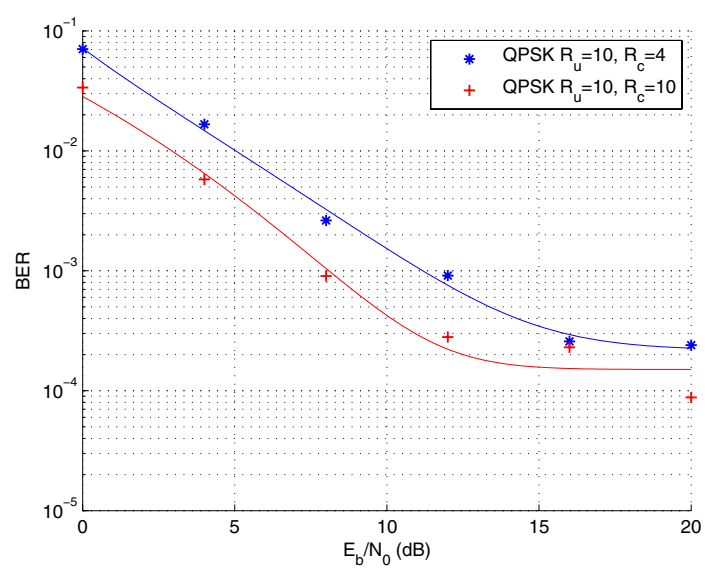

Fig. 10. QPSK for degraded cooperation links

equipped with non directive antennas) of a satellite DVB-S2 transmission. The idea is to build a cooperation among a set of mobile terminals, in a way that the signal received by each single device is the result of the composition of more replicas of the same signal sent by other cooperating devices.

The choice of the adopted link cooperation method (Amplify and Forward) has been suggested by the satellite operational context (Figure 1), characterized by unbalanced link strengths and limited complexity available at cooperators.

Link budget analysis shows that by choosing feasible system parameters (satellite spot power, co-operation amplification factor, number of co-operating terminals, terminal power dedicated to co-operation) we obtain signal-to-noise ratios compatible with DVB-S down-link profiles for up to 16-APSK constellations.

Under a more realistic scenario, where all the cooperators are independently faded accordingly to the Corazza-Vatalaro channel model, high order modulations are still possible in presence of favorable propagation conditions.

Link cooperation enables the reception of DVB-S2 services from handheld terminals when a cluster of cooperating users is present. This is a common context when professional users are involved (emergency rescue teams, tactical scenarios).

In the case of personal communications, the link cooperation technique may be offered as an option to overcome reception limitation, so the single subscriber has the possibility to choose if to participate to the cluster or not. This model allows the user to retain the control over the power resources of its terminal.

\section{REFERENCES}

[1] ETSI TR 102376 V1.1.1 (2005-02) "Digital Video Broadcasting (DVB): User guidelines for the second generation system for Broadcasting, Interactive Services, News Gathering and other broadband satellite applications (DVB-S2),".

[2] ETSI EN 302307 V1.1.1 (2004-06) "Digital Video Broadcasting (DVB); Second generation framing structure, channel coding and modulation systems for Broadcasting, Interactive Services, News Gathering and other broadband satellite applications.".

[3] Richharia M. "Mobile Satellite Communications: Principles and Trends". Addison-Wesley, ISBN 020133142 X
[4] Sendonaris A., Erkip E., and Aazhang B. "User cooperation diversity part I: system description". IEEE Transactions on Communications, vol. 51, pp. 1927-1938, Nov. 2003.

[5] Sendonaris A., Erkip E., and Aazhang B. "User cooperation diversity - part II: Implementation Aspects and Performance Analysis". IEEE Transactions on Communications, vol. 51, pp. 1939-1948, Nov. 2003.

[6] J. Nicholas Laneman "Cooperative Diversity in Wireless Networks: Algorithms and Architectures". PhD Thesis, Massachusetts Institute of Technology, Cambridge MA, Sep. 2002.

[7] Ribeiro, A. and Giannakis G. "Fixed and Random Access Cooperative Networks". EURASIP NEWSLETTER, invited article - submutted january $18,2006$.

[8] Ribeiro, A. and Cai X. and Giannakis G. "Symbol Error Probabilities for General Cooperative Links". IEEE Transactions on wireless communications, 4(3):1264-1273, 2005.

[9] F. Vatalaro, G. Corazza "Probability of Error and Outage in a RiceLognormal Channel for Terrestrial and Satellite Personal Communications". IEEE Transactions on Communications, vol. 44, pp. 921-924, Aug. 1996.

[10] F. Vatalaro G. Corazza C. Caini C. Ferrarelli "Analysis of LEO, MEO, and GEO Global Mobile Satellite Systems in the Presence of Interference and Fading". IEEE Journal on selected areas in communications, vol. 13, pp. 291-300, Feb. 1995.

[11] G. Corazza, F. Vatalaro "A Statistical Model for Land Mobile Satellite Channels and Its Applications to Nongeostationary Orbit Systems". IEEE Transactions on vehicular technology, vol. 43, pp. 738-741, Aug. 1994. 